\title{
Umbilical Cord Lining Membrane and Wharton's Jelly-Derived Mesenchymal Stem Cells: the Similarities and Differences
}

\author{
Marc G. Jeschke ${ }^{1,2,3}$, Gerd G. Gauglitz ${ }^{4}$, Thang T. Phan ${ }^{5}$, David N. Herndon ${ }^{6,7}$ and Katsuhiro Kita ${ }^{*}, 6$ \\ ${ }^{1}$ Ross Tilley Burn Centre, Sunnybrook Health Sciences Centre, Toronto, Ontario, M4N 3M5, Canada \\ ${ }^{2}$ Department of Surgery, University of Toronto, Ontario M4N 3M5, Canada \\ ${ }^{3}$ Sunnybrook Research Institute, Toronto, Ontario M4N 3M5, Canada \\ ${ }^{4}$ Department of Dermatology and Allergology, Ludwig Maximilians University, Munich, Germany \\ ${ }^{5}$ Department of Surgery, Yong Loo Lin School of Medicine and Centre for Craniofacial \& Regenerative Biology, \\ National University of Singapore and Cell Research Corp. Pte, Lte, Singapore \\ ${ }^{6}$ Department of Surgery and Shriners Hospitals for Children, The University of Texas Medical Branch, Galveston, TX \\ 77550, USA \\ ${ }^{7}$ Department of Pediatrics, The University of Texas Medical Branch, Galveston, TX 77550, USA
}

\begin{abstract}
The umbilical cord tissue has gained attention in recent years as a source of multipotent cells. Due to its widespread availability, the umbilical cord may be an excellent alternative source of cells for regenerative medicine. Anatomically, umbilical cord tissue is constituted of several different parts, and, accordingly, immunostaining of cord tissue sections revealed differential distribution of several markers and extracellular matrix, distinguishing the various layers. Wharton's jelly is the major component filling the inner part of the umbilical cord tissue, and it has been commonly used as a source of obtaining multipotent cells from umbilical cord. We recently reported isolating mesenchymal stem cells from cord lining membrane (sub-amnion). However, because of several anatomically distinct zones found in the umbilical cord, isolated multipotent cells sometimes show heterogeneity. In addition, differences in isolation technique may lead to further variation. In this review, we discuss the similarities and differences between the cells derived from each sub-region, including sub-amnion as recently reported by us. We further explore the specific features and advantages/disadvantages of Wharton's jelly and the other sub-compartments in the umbilical cord tissue as sources of stem cells/multipotent cells.
\end{abstract}

Keywords: Wharton's jelly, umbilical cord, mesenchymal stem cells, sub-amnion.

\section{INTRODUCTION}

Since Pittenger and colleagues demonstrated the successful isolation of multipotent mesenchymal stem cells (MSCs) from human bone marrow [1], it has been considered the primary source from which to obtain MSCs; those derived from bone marrow are the most studied and well-documented. Although it is well established, bone marrow still has some marked disadvantages as a source of MSCs. First, only a very small fraction $(0.05-0.001 \%)$ of total bone marrow cells contains MSCs [1]. Second, collection of bone marrow requires a very painful and invasive procedure. Thus, the search for alternative sources of MSCs continues.

Adipose tissue [2] and amniotic fluid [3] have recently come onto the scene as alternative sources. Despite its plentiful nature, an invasive procedure is still required to collect adipose tissues [2]. Conversely, umbilical cord and amniotic membrane represent fascinating alternative sources

*Address correspondence to this author at the Department of Biochemistry and Molecular Biology, University of Texas Medical Branch, Mail route 0647, Basic Science Building, 301 University Boulevard, Galveston, Texas 77555; Fax: 409-772-2880; E-mail: kakitatemp@yahoo.com due to several reasons: 1) collection of these tissues poses fewer ethical issues since these tissues are usually discarded as biological waste 2) the tissues are available world-wide, and thus it would promote MSC-based research all across the globe. Although there are over a dozen original research articles reporting isolation of MSCs from umbilical cord [410] and amniotic membrane [11], there are considerable differences between research groups in terms of characteristics (mainly expression of particular markers) of the MSCs obtained. Such differences may originate from the skills and the methods that each research group applies.

In this review, we will focus on the types of MSCs that can be isolated from the umbilical cord tissue. In particular, we will compare and contrast MSCs derived from two subregions (i.e., sub-amnion and Wharton's jelly) of the tissue. As a supplement to this discussion, we also encourage readers to peruse a very excellent review by Troyer and Weiss [12].

\section{ANATOMY OF THE UMBILICAL CORD TISSUE}

Before discussing the differences between MSCs obtained from the umbilical cord tissue, we should take a 


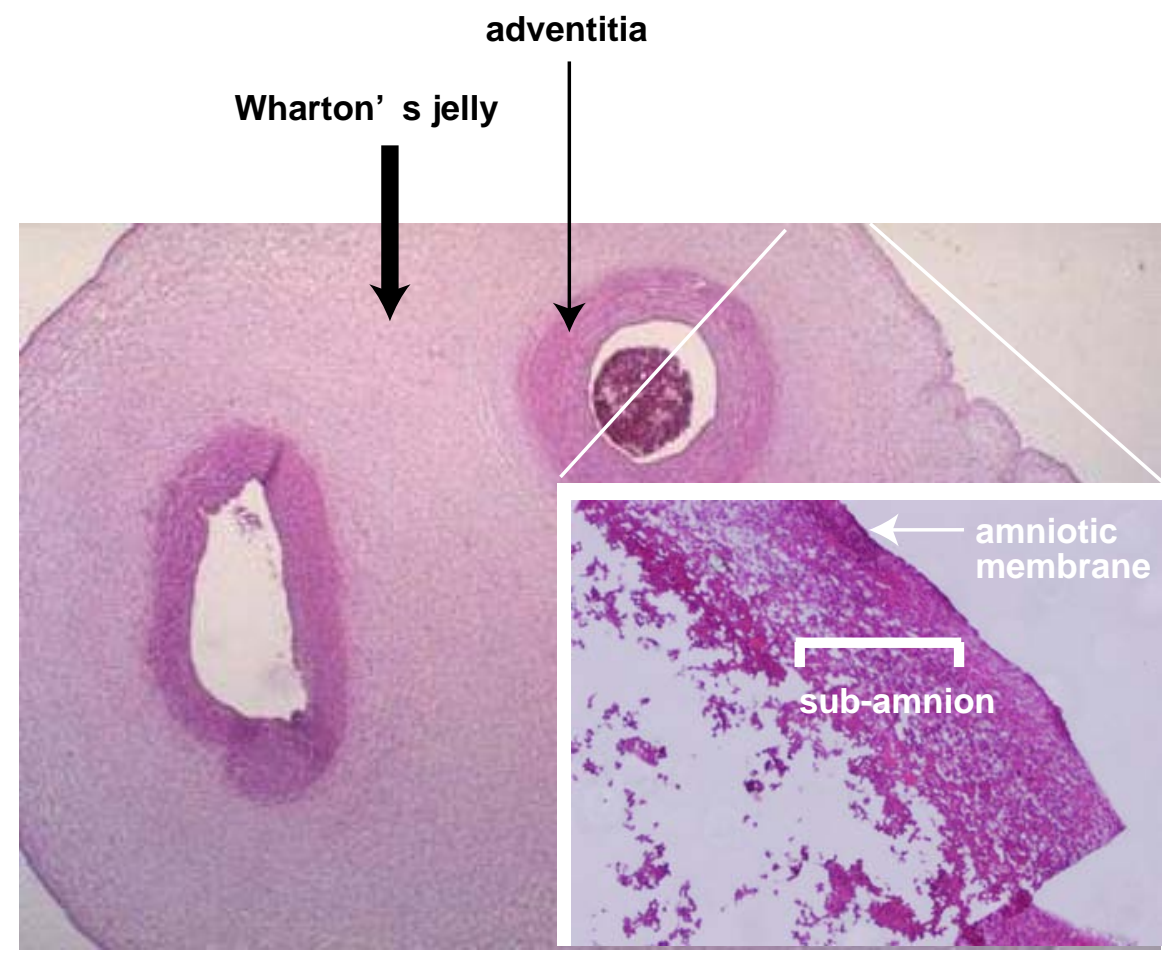

Fig. (1). Schematic illustration of human umbilical cord.

deeper look into the architecture of the tissue. Fig. (1) illustrates a schematic cartoon of human umbilical cord. Umbilical cord consists out of: 1) amniotic membrane 2) sub-amnion 3) Wharton's jelly 4) adventitia and 5) media (umbilical cord blood vessels). Amniotic membrane (amniotic epithelium) is the outer envelop of the tissue, and it represents the only epithelium in the tissue. Thus, this layer is not a source of MSCs. However, it should be noted that Miki et al., reported multipotent epithelial SCs isolated from amniotic membrane [11].

Sub-amnion, Wharton's jelly, and adventitia do not have clearly visible structural borders among them, although Wharton's jelly is characterized by a relatively lower cell density than the other layers. Cells are loosely arranged around the large spaces in Wharton's jelly. Also, these spaces between cells do not contain collagens, instead, proteoglycans are enriched here. These proteoglycan-rich spaces may function as reservoirs to supply nutrients to the cells in Wharton's jelly [13], and they may also help give flexibility and strength to the umbilical cord. In addition to these matrices filling Wharton's jelly, blood vessels are located near the center of the tissue. Two blood vessels are found. These vessels are continuous between a mother and her baby and are surrounded by very strong, elastic musclelike tissues (adventitia), mainly constituted of muscle cells to mechanically support the blood vessels.

Although structurally consecutive, histological analyses and immunostaining of collagens indicate that sub-amnion, Wharton's jelly, and adventitia are distinct regions. Furthermore, $\alpha$-smooth muscle actin and smooth muscle myosin are exclusively expressed in sub-amniotic region and adventitia. Another interesting fact is that sub-amnion and the juxtavascular region (adventitia) exclusively express
MIB-1 (proliferation marker) [14], a detail that will be discussed further in section 3 .

\section{WHARTON'S JELLY-DERIVED CELLS}

Searching "Wharton's jelly" in PubMed through 2010 returns 244 publications. Many of these research reports have described isolation or characterization of actual MSCs. Meanwhile, the term "MSCs" has sometimes been used as an acronym for different words, e.g., Marrow (or Multipotent) Stromal Cells. Thus, readers could become confused as to whether the term "MSCs" defines either "mesenchymal stem cells" or "marrow stromal cells". MSC may even be short for Matrix Stem Cell on occasion [6]. Therefore, we wish to clearly express that, in this review, MSCs will be used as an acronym for "mesenchymal stem cells" or "matrix mesenchymal stem cells". The minimum criteria of "mutipotent stromal cells" are concisely summarized by Dominici et al., and we encourage reading this paper [15]. Although MSC and "multipotent stromal cell" should be distinguished, we believe that the guidelines proposed by Dominici et al., [15] would also be very useful to define MSCs. In addition, MSCs may express some primitive markers not listed in this proposal. Davies' group reported the isolation and characterization of human umbilical cord perivascular (HUCPV) cells [5]. Although HUCPV cells share the similarity with Wharton's jelly- and sub-amnion-derived MSCs in terms of marker molecules (for example, CD73, CD105, CD90, and CD44), HUCPV cells are pericyte marker positive, and the cells showed bone node formation [5].

In addition to the definition of cells, another issue that may stir the field is the heterogeneity of cells obtained from Wharton's jelly, which is most likely due to the methods and 
skill level of each researcher. The nature of Wharton's jelly is also accountable. To isolate the Wharton's jelly, the tissues are digested with proteases or minced with a grinder [16]. It is imperative that the blood vessels and umbilical cord epithelium be removed prior to this process. However, it is possible that some reports may use the whole umbilical cord, and this may account for some heterogeneity. If so, due diligence to the dissection and preparation techniques employed should lead to more homogenous populations of Wharton-derived MSCs.

Thus, quality control of MSCs isolated from Wharton's jelly is probably more difficult than cord lining MSCs (CLMSCs) due to the aforementioned considerations. At the same time, Wharton's jelly presents undeniable advantages such as the ability to obtain larger quantity of cells within relatively short periods of time. If the quality of MSCs is not crucial to the application, digestion/mincing of the whole umbilical cord tissue may be an alternative choice to obtain large amounts of MSCs mainly from Wharton's jelly. For semi-industrial scale preparation of MSCs, this may be a reasonable option. Interestingly, Majore et al., showed counterflow centrifugal elutriation, a classic method that allows gentle large-scale isolation of cells [17], as an effective strategy to enrich primitive stem cells from umbilical cord-derived cell fractions [18]. Thus, this method appears to be a suitable option to combine with crude cell populations obtained from Wharton's jelly if needed.

\section{SUB-AMNION-DERIVED CELLS}

We have previously attempted to isolate MSCs from subamnion (cord lining membrane) of the umbilical cord [19]. Strictly speaking, the term "cord lining membrane" may specifically refer to the epithelium of the umbilical cord. Therefore, instead, we will continue using "sub-amnion" throughout the rest of this review. To obtain MSCs from sub-amnion with minimum contamination of other cell types, the inner part of the cord lining membrane should be removed with a razor blade, resulting in a thin layer of subamnion. Since it is probably impossible to completely remove the adjacent region underneath the epithelium, dissection with a razor blade would leave a thin layer of "mixed" tissue. Based on our experience, these thin membranes exclusively contain cells from amnion epithelium and sub-amnion. Thus, we believe it is not difficult to preclude the contamination of Wharton's jelly-derived cells using this method of dissection.

However, the major disadvantage associated with isolation of CL-MSC is the extremely time-consuming nature of the process. In our experience, there are at least 2 "working" days after receiving the tissues. Day 1 consists of extensive rinsing and opening of the tissues followed by more extensive rinsing, and the opened tissues are incubated in serumfree medium (plus double concentration of antibiotics) for a day or two. Day 2 requires working in a cell culture cabinet for several hours to prepare thin pieces of tissues. In addition to these working days, there is an extensive wait time while the MSCs migrate from the pieces of tissue (usually $\sim 3$ weeks). Hence, in terms of required time, CL-MSCs may not be suitable for rapid, large-scale preparation of cells. We have not compared the quantity of MSCs available from sub- amnion and Wharton's jelly, although it seems reasonable to conclude that greater quantities of cells are available from Wharton's jelly.

While CL-MSCs are more difficult to isolate in comparison to Wharton's jelly cells, they may possess one very important advantageous quality - a greater proliferative capacity. As mentioned in section 1, immunohistochemistry of the umbilical cord tissue revealed that sub-amnion and the juxtavascular position (adventitia) were exclusively stained with anti-MIB-1 antibody, while the Wharton's jelly was not [14]. MIB-1 is a proliferation marker. Taken together with the data showing great proliferative capability of CL-MSCs confirmed by the colony-forming assay [19], this evidence implies that CL-MSCs may be superior to Wharton's jellyderived MSCs in terms of potential in ex vivo expansion.

\section{COMPARISON OF CELL SURFACE AND INTRA- CELLULAR MARKERS}

The comparison of Wharton's jelly-derived and CL-MSC cell surface markers is summarized in Table 1. Prominent

Table 1. Comparison of Cell Surface and Intracellular Markers between CL- and Wharton's Jelly-MSCs. +, Positive; -, Negative; -/+, Essentially Negative, but Some Report(s) Showing Positive; NA, Not Clear Yet

\begin{tabular}{|c|c|c|}
\hline Molecules & CL-MSC & Wharton's Jelly-MSC \\
\hline FGF-4 & $-[19]$ & NA \\
\hline TERT & $-[19,22]$ & $\mathrm{NA} /-[20]$ \\
\hline Sox-2 & $-/+[19,22]$ & $\mathrm{NA} /+/-[6,20]$ \\
\hline Rex-1 & $-/+[19,22]$ & $\mathrm{NA} /-[20]$ \\
\hline Oct-4 & $+[19,22]$ & $-/+[6,24]$ \\
\hline Nanog & $+[19,22]$ & $+/-[6,20]$ \\
\hline c-Kit & + (weak) [19] & $-/+[24]$ \\
\hline CD34 & $-[19]$ & $-[6]$ \\
\hline CD45 & $-[19]$ & $-[6]$ \\
\hline SSEA-1 & $+[19]$ & $\mathrm{NA} /+[20]$ \\
\hline SSEA-4 & $+[19]$ & $\mathrm{NA} /+[20]$ \\
\hline TRA1-60 & + (weak) [19] & $\mathrm{NA} /+[20]$ \\
\hline TRA1-81 & + (weak) [19] & $\mathrm{NA} /+[20]$ \\
\hline TRA2-54 & + (weak) [19] & NA \\
\hline MHC class I & $+[19,22]$ & $+[6]$ \\
\hline MHC class II & $-[19,22]$ & $-[6]$ \\
\hline CD29 & $+[19]$ & $+[6]$ \\
\hline $\mathrm{CD} 44$ & $+[19]$ & $+[6]$ \\
\hline CD90 & $+[19]$ & $+[6]$ \\
\hline CD73 & $+[19,22]$ & $+[6]$ \\
\hline CD105 & $+[19,22]$ & $+[24]$ \\
\hline $\mathrm{CD} 23$ & $+[19]$ & NA \\
\hline STRO-1 & $-[19]$ & $-[38]$ \\
\hline CD31 & $-[19]$ & NA [24] \\
\hline CD146 & $+[19]$ & $+[38]$ \\
\hline $\mathrm{CD} 10$ & $+[19]$ & $+[6]$ \\
\hline CD13 & $+[19]$ & $+[6]$ \\
\hline CD14 & $+[19]$ & $-[6]$ \\
\hline
\end{tabular}




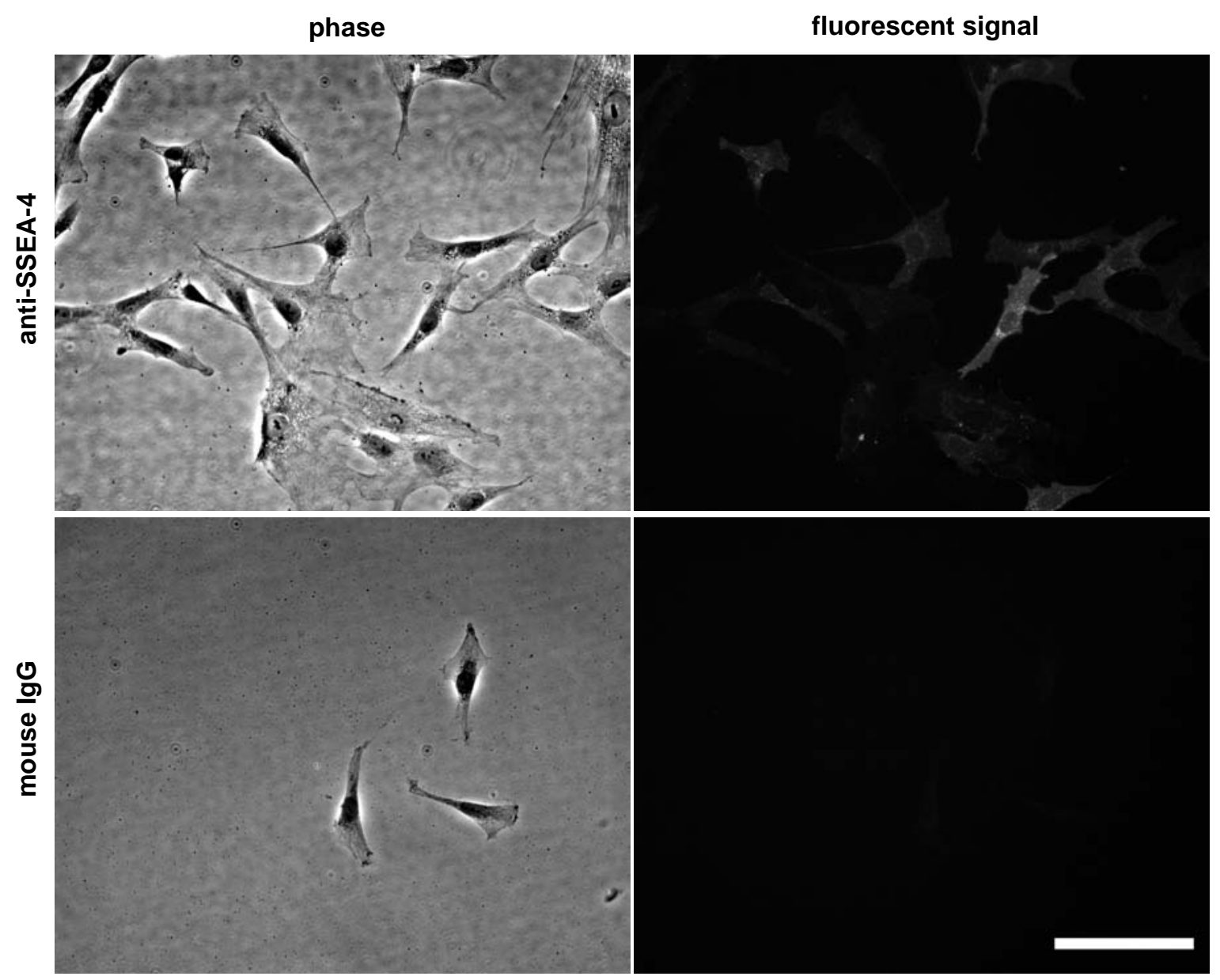

Fig. (2). Example of SSEA-4 immunofluorescence staining. Note that SSEA-4 expression may be high in small, well-growing cells, which are often referred as "spindleshape" cells.

differences include several surface markers expressed in embryonic stem cells (SSEA and TRA antigens). These markers are included in the criteria for embryonic SC classification but not for MSCs. Although the expression of TRA antigens was very week, SSEA antigens were relatively well expressed on CL-MSCs, particularly SSEA-4 (see immunostaining of SSEA-4, Fig. (2)). Furthermore, the number of SSEA-4 positive cells was also high in isolated MSC populations. In contrast, Wharton's jelly-derived MSCs have not been found to express these markers, barring a couple of recent reports [20, 21]. Interestingly, flat, large cells, which are sometimes referred as "aged" MSCs by the other groups [8], may express lower level of SSEA-4 (Fig. 2).

Regarding MSC markers, there is probably no appreciable difference. Furthermore, other than the two discussed above, MSCs from both origins do not express embryonic SC markers, such as TERT, Rex-1, Sox-2, FGF-4, although we recently found that CL-MSCs expressed Rex-1 and Sox-2 [22]. In our first report, we could not detect both Rex-1 and Sox-2 mRNA [19]. This discrepancy may be due to the differences of primer sets used in each study. We also cannot preclude that both molecules are easily lost, and the detection of Rex-1 and Sox-2 may rely on technical issues. Expression of these two embryonic SC markers can be awkward, although we should note that CL-MSCs do not form tumors in nude mice [23] and do not grow in soft agar [19]. Oct-4 and Nanog expression differs among MSCs [19, $22,24]$, the significance of which remains unclear. Although these two molecules are defined as important in maintaining multipotency of embryonic SCs, neither molecule is included in the criteria of MSCs [15]. MSCs without Oct-4 and Nanog still retain the capacity to differentiate into several different lineages $[4,7,8]$. Expression of Oct-4 and Nanog may reflect superior capability of MSCs in terms of multipotency, however, both molecules may be negligible in terms of multipotency of MSCs from umbilical cord.

Minimum criteria of MSCs include differentiation capacity toward three lineages (adipogenic, osteogenic, and chondrogenic). Notably, in addition to these three lineages, Wharton's jelly-derived MSCs have been shown to differentiate into other cell types, such as neuronal [7,8], endothelial [25], and cardiogenic [4] lineages. Recent studies also demonstrated derivation of hepatocytes from umbilical cord epithelium [11]. We have not systematically compared differentiation capacity of cells from sub-amnion/amnion epithelium and Wharton's jelly. However, judging from accumulating evidence, it is not clear whether there are significant differences between sub-amnion- and Wharton's 
jelly-originated MSCs. Interestingly, a recent report by Ishige et al. showed that Wharton's jelly MSCs were less effective precursor in terms of osteogenic potential [26].

We confirmed that CL-MSCs did not express significant level of CD31 (endothelial marker) [19]. Other groups have corroborated our findings [22]. We think it is particularly important to investigate this marker in Wharton's jelly MSCs, because Wharton's jelly may very well be contaminated with endothelial cells derived from blood vessels of the umbilical cord tissue. If the tissues are grinded during isolation of MSCs, this contamination of endothelial cells may be inevitable, though minor populations of contaminated cells may disappear after culturing for several passages. In fact, Wharton's jelly-derived matrix cells were reported to be CD31 positive [6], however, the other study and a review reported Wharton's jelly cells did not express CD31 $[24,27]$.

The most outstanding feature of CL-MSCs is the expression of CD14 [19]. CD14 is a low affinity LPS recaptor [28], and is widely recognized as a common marker for macrophages. However, CL-MSCs express negligible levels of other hematopoietic markers, CD34 and CD45 [19]. In addition, CD14 expression was reported in cell types other than macrophages, so it may not necessarily be an identifying characteristic as once thought [29]. Thus, although we cannot preclude the possibility that CL-MSCs are derived from small fractions of hematopoietic SCs con-taminating sub-amnion, this does not appear to be the case when considering other markers, and it is also not a unique concept that CL-MSCs are non-hematopoietic cells expres-sing CD14. Conversely, to our knowledge, no Wharton's jellyderived MSCs express CD14. The function and significance of CD14 expression on CL-MSCs has yet to be determined, but it is interesting to note that the soluble form of CD14 can down regulate $T$ cell activation [30]. Currently the study is ongoing to further investigate the functional significance of CD14 in CL-MSCs. It is rather exciting to speculate that CLMSCs could use CD14 as a method of modulating immune cells, which may make them particularly attractive candidates for tissue regeneration and transplant therapeutic applications. Most recent report by La Rocca et al., also described the expression of CD68, the other macrophage marker [31]. As the authors suggested in the report, it is very interesting if the expression of such atypical markers contribute to hypoimmunogenic and tolerogenic nature of MSCs.

\section{COMPARISON OF CYTOSKELETAL MARKERS AND EXTRACELLULAR MATRICES}

Cytoskeletal components are sometimes used to infer the origin of cells from the various components of umbilical cord tissue. For example, $\alpha$-smooth muscle actin seems to be commonly expressed in both Wharton's jelly- and CL-MSCs $[8,19]$. Vimentin is highly expressed in CL-MSCs [19], and probably similarly expressed in Wharton's jelly MSCs as well [8]. Desmin was not expressed in CL-MSCs [19]. Since the tissue section showed some desmin staining [8], this may differ between CL-MSCs and Wharton's jelly MSCs. Alternatively, desmin expression may be lost after isolation of cells. Strong cytokelatin expression may be a hallmark of aged MSCs as sometimes reported [8]. In contrast to this report, CL-MSCs expressed only trace amounts of cytokelatin [19], which, interestingly, may indicate CL-MSCs as precursors of the umbilical cord epithelium or epithelial SC [11].

Alaminos et al., referred to E-cadherin in their paper as an undifferentiation marker of MSCs [21]. Likewise, it is known that "embryonic" stem cells express E-cadherin [32]. However, it is well established in cell biology that fibroblasts express very little E-cadherin [33]. Furthermore, it is widely accepted that decreased E-cadherin expression correlates with the epithelial-mesenchymal transition [34]. Taken together, these observations are conflicting, and the expression of E-cadherin in MSCs seems unlikely. We propose that, since careful and proper choice of antibodies is very important in generating interpretable data, E-cadherin positive cells demonstrated in this article may be caused by inadequate methods or low quality of antibodies used in the study [21].

In short, cytoskeletal elements are apparently the same between CL-MSCs and Wharton's jelly MSCs.

\section{SIMILARITY WITH BONE MARROW-DERIVED MSCS}

Bone marrow MSCs are likely to share similar characteristics with CL-MSCs and Wharton's jelly MSCs, although there are some minor differences. STRO-1 [35], which is sometimes used to enrich bone marrow MSCs from a mixed population [36], is expressed in neither CL-MSCs nor Wharton's jelly MSCs. However, a lack of STRO-1 is also reported in some bone marrow MSCs [37], therefore, this marker expression seems to be variable between researchers and antibodies used for detection. CD23 expression was reported for bone marrow MSCs in Pittenger's publication [1], and our studies showed that CL-MSCs are also CD23 positive. To our knowledge, CD23 expression has not been clearly documented in studies characterizing MSCs from Wharton's jelly.

As mentioned in section 4, it has been shown that Wharton's jelly MSCs are less effective precursor in terms of osteogenic potential [26]. Since bone marrow MSCs can differentiate into the osteogenic lineage well [1], Wharton's jelly MSCs and bone marrow MSCs may demonstrate disparate gene and protein expression profiles regarding osteogenesis.

\section{CONCLUDING REMARKS}

CL-MSCs likely hold excellent potential in terms of their expansion capacity and possibly multipotency. Although Wharton's jelly-derived MSCs may show more variation in terms of quality of cells, Wharton's jelly is still a very useful part of the umbilical cord tissue from which to obtain large quantity of cells. Studies proving that Wharton's jelly is an adequate source of MSCs continue to emerge.

Rather than debating which anatomic region of the umbilical cord is "better" for isolating MSCs, we suggest that the researcher make the choice that is right for them, 
depending on the quantity and quality required for each specific application.

\section{ACKNOWLEDGEMENTS}

This work was supported by Anderson Foundation for Research and Clayton Foundation. The authors also acknowledge Dr. Hal K. Hawkins for his comments (Department of Pathology, University of Texas Medical Branch) regarding the definition of sub-compartments in the umbilical cord tissue. We also thank Dr. Celeste C. Finnerty (Department of Surgery, University of Texas Medical Branch) and administrative staff for their dedicated assistance.

\section{CONFLICT OF INTEREST}

Dr. Phan is the founder and a shareholder of Cell Research Corporation.

\section{ABBREVIATIONS}

CL-MSC = Cord lining membrane mesenchymal stem cell

MSC = Mesenchymal stem cell

\section{REFERENCES}

[1] Pittenger MF, Mackay AM, Beck SC, et al. Multilineage potential of adult human mesenchymal stem cells. Science 1999; 284: 143-7.

[2] Bunnell BA, Flaat M, Gagliardi C, Patel B, Ripoll C. Adiposederived stem cells: Isolation, expansion and differentiation. Methods 2008; 45: 115-20.

[3] Coppi PD, Georg BJ, Siddiqui MM, et al. Isolation of amniotic stem cell lines with potential for therapy. Nat Biotechnol 2007; 25: 100-6.

[4] Wang H-S, Hung S-C, Peng S-T, et al. Mesenchymal stem cells in the Wharton's jelly of the human umbilical cord. Stem Cells 2004; 22: 1330-7.

[5] Sarugaser R, Lickorish D, Baksh D, Hosseini MM, Davies JE. Human umbilical cord perivascular (HUCPV) cells: a source of mesenchymal progenitors. Stem Cells 2005; 23: 220-9.

[6] Weiss ML, Medicetty S, Bledsoe AR, et al. Human umbilical cord matrix stem cells: preliminary characterization and effect of transplantation in a rodent model of Parkinson's disease. Stem Cells 2006; 24: 781-92.

[7] Lu L-L, Liu Y-J, Yang S-G, et al. Isolation and characterization of human umbilical cord mesenchymal stem cells with hematopoiesissupportive function and other potentials. Haematologica 2006; 91: 1017-26.

[8] Karahuseyinoglu S, Cinar O, Kilic E, et al. Biology of stem cells in human umbilical cord stroma: in situ and in vitro surveys. Stem Cells 2007; 25: 319-31.

[9] Lund RD, Wang S, Lu B, et al. Cells isolated from umbilical cord tissue rescue photoreceptors and visual functions in a rodent model of retinal disease. Stem Cells 2007; 25: 602-11.

[10] Rocca GL, Anzalone R, Corrao S, et al. Isolation and characterization of Oct- $4^{+} / \mathrm{HLA}-\mathrm{G}^{+}$mesenchymal stem cells from human umbilical cord matrix: differentiation potential and detection of new markers. Histochem Cell Biol 2009; 131: 267-82.

[11] Miki T, Lehmann T, Cai H, Stolz DB, Strom SC. Stem cell characteristics of amniotic epithelial cells. Stem Cells 2005; 23: 1549-59.

[12] Troyer DL, Weiss ML. Concise Review: Wharton's jelly-derived cells are a primitive stromal cell population. Stem Cells 2008; 26: 591-9.

[13] Sobolewski K, Małkowski A, Bańkowski E, Jaworski S. Wharton's jelly as a reservoir of peptide growth factors. Placenta 2005; 26 : $747-52$.
[14] Nanaev AK, Kohnen G, Milovanov AP, Domogatsky SP, Kaufmann P. Stromal differentiation and architecture of the human umbilical cord. Placenta 1997; 18: 53-64.

[15] Dominici M, Blanc KL, Mueller I, et al. Minimal criteria for defining multipotent mesenchymal stromal cells. The International Society for Cellular Therapy position statement. Cytotherapy 2006; 8: 315-7.

[16] Seshareddy K, Troyer D, Weiss ML. Methods to isolate mesenchymal-like cells from Wharton's jelly of umbilical cord. Methods Cell Biol 2008; 86: 101-19.

[17] Lutz MP, Gaedicke G, Hartmann W. Large-scale cell separation by centrifugal elutriation. Anal Biochem 1992; 200: 376-80.

[18] Majore I, Moretti P, Hass R, Kasper C. Identification of subpopulations in mesenchymals stem cell-like cultures from human umbilical cord. Cell Commun Signal 2009; 7: 6.

[19] Kita K, Gauglitz GG, Phan TT, Herndon DN, Jeschke MG. Isolation and characterization of mesenchymal stem cells from the sub-amniotic human umbilical cord lining membrane. Stem Cells Dev 2010; 19: 491-502.

[20] Fong C-Y, Richards M, Manasi N, Biswas A, Bongso A. Comparative growth behaviour and characterization of stem cells from human Wharton's jelly. Reprod Biomed Online 2007; 15: 708-18.

[21] Alaminos M, Pérez-Köhler B, Garzón I, et al. Transdifferentiation potentiality of human Wharton's jelly stem cells towards vascular endothelial cells. J Cell Physiol 2010; 223: 640-7.

[22] Reza HM, Ng B-Y, Phan TT, et al. Characterization of a novel umbilical cord lining cell with CD227 positivity and unique pattern of P63 expression and function. Stem Cell Rev 2010: in press.

[23] Sivalingam J, Krishnan S, Ng WH, et al. Biosafety assessment of site-directed transgene integration in human umbilical cord-lining cells. Mol Ther 2010; 18: 1346-56.

[24] Can A, Karahuseyinoglu S. Concise Review: Human umbilical cord stroma with regard to the source of fetus-derived stem cells. Stem Cells 2007; 25: 2886-95.

[25] Wu KH, Zho B, Lu SH, et al. In vitro and In Vivo Differentiation of human umbilical cord derived stem cells into endothelial cells. J Cell Biochem 2007; 100: 608-16.

[26] Ishige I, Nagamura-Inoue T, Honda MJ, et al. Comparison of mesenchymal stem cells derived from arterial, verous, and Wharton's jelly explants of human umbilical cord. Int J Hematol 2009; 90: 261-9.

[27] Conconi MT, Burra P, Liddo RD, et al. CD105(+) cells from Wharton's jelly show in vitro and in vivo myogenic differentiative potential. Int J Mol Med 2006; 18: 1089-96.

[28] Wright SD, Ramos RA, Tobias PS, Ulevitch RJ, Mathison JC. CD14, a receptor for complexes of lipopolysaccharide (LPS) and LPS binding protein. Science 1990; 249: 1431-3.

[29] Stoll LL, Denning GM, Li W-G, et al. Regulation of endotoxininduced proinflammatory activation in human coronary artery cells: expression of functional membrane-bound CD14 by human coronary artery smooth muscle cells. J Immunol 2004; 173: 133643.

[30] Nores JER, Bensussan A, Vita N, et al. Soluble CD14 acts as a negative regulator of human $\mathrm{T}$ cell activation and function. Eur $\mathrm{J}$ Immunol 1999; 29: 265-76.

[31] Rocca GL, Anzalone R, Farina F. The expression of CD68 in human umbilical cord mesenchymal stem cells: new evidences of presence in non-myeloid cell types. Scand J Immunol 2009; 70: 161-2.

[32] Bloor DJ, Metcalfe AD, Rutherford A, Brison DR, Kimber SJ Expression of cell adhesion molecules during human preimplantation embryo development. Mol Hum Reprod 2002; 8: 237-45.

[33] Nose A, Nagafuchi A, Takeichi M. Expressed recombinant cadherins mediate cell sorting in model systems. Cell 1988; 54: 993-1001.

[34] Cavallaro U, Christofori G. Cell adhesion and signalling by cadherins and Ig-CAMs in cancer. Nat Rev Cancer 2004; 4: 11832 .

[35] Simmons PJ, Torok-Storb B. Identification of stromal cell precursors in human bone marrow by a novel monoclonal antibody, STRO-1. Blood 1991; 78: 55-62. 
[36] Gronthos S, Zannettino ACW, Hay SJ, et al. Molecular and cellular characterisation of highly purified stromal stem cells derived from human bone marrow. J Cell Sci 2003; 116: 1827-35.

[37] Colter DC, Sekiya I, Prockop DJ. Identification of a subpopulation of rapidly self-renewing and multipotential adult stem cells in colonies of human marrow stromal cells. Proc Natl Acad Sci USA 2001; 98: 7841-5.

[38] Baksh D, Yao R, Tuan RS. Comparison of proliferative and multilineage differentiation potential of human mesenchymal stem cells derived from umbilical cord and bone marrow. Stem Cells 2007; 25 : 1384-92

Received: March 13, 2011

Revised: May 17, 2011

Accepted: June 12, 2011

(C) Jeschke et al.; Licensee Bentham Open.

This is an open access article licensed under the terms of the Creative Commons Attribution Non-Commercial License (http://creativecommons.org/ licenses/by-nc/3.0/), which permits unrestricted, non-commercial use, distribution and reproduction in any medium, provided the work is properly cited. 